Original Article

\title{
ENHANCING DISSOLUTION RATE OF INDOMETHACIN BY IN SITU CRYSTALIZATION; DEVELOPMENT OF ORALLY DISINTEGRATING TABLETS
}

\author{
NADIA H. NAIEM*, EBTESSAM A. ESSA, GAMAL M. ELMAGHRABY \\ College of Pharmacy, University of Tanta, Pharmaceutical Technology, Department, Tanta University, Egypt \\ Email: nadia.naiem@yahoo.com
}

Received: 16 Dec 2017 Revised and Accepted: 22 Mar 2018

\section{ABSTRACT}

Objective: The main objective of this study was to investigate the potential of in situ crystallization of indomethacin, in presence or absence of hydrophilic materials, to improve drug dissolution with the goal of developing fast disintegrating tablets.

Methods: Indomethacin crystals were prepared by bottom up approach. Water containing hydrophilic additive (polymer or/and surfactant) was added to ethanolic solution of indomethacin while stirring. The selected polymers were hydroxylpropylmethyl cellulose E5 (HPMC E5), polyethylene glycol 6000 (PEG6000) and polyvinylpyrrolidone K40 (PVP K40). The surfactants used were Tween80 and Glucire 44/14. The precipitated particles were collected and air dried. Solid state characterization were performed in addition to in vitro release studies in both acidic (0.1 N HCL) and alkaline medium (phosphate buffer pH 6.8). Optimized formulation was selected to develop fast disintegrating tablets.

Results: Thermal behavior suggested modulation in crystalline nature with reduction in particle size that was confirmed by X-ray diffraction results. Infrared spectroscopy excluded any interaction between drug and hydrophilic excipients. Drug dissolution in acid media showed slight improve in drug release, while marked increase was observed in the alkaline media. Combination between Tween80 and HPMC (F7) showed the best dissolution parameters with 5-folds enhancement in release efficiency (RE) compared to pure drug. Formula F7 was successively used to formulate fast disintegrating tablets with prompted release of $58 \%$ of the loaded dose and RE of $83 \%$.

Conclusion: In situ crystallization of indomethacin is a good approach for enhanced dissolution rate with the presence of hydrophilic additives during precipitation process improving the efficiency.

Keywords: In situ crystallization, Enhance solubility, Indomethacin, Fast disintegrating tablets

(C) 2018 The Authors. Published by Innovare Academic Sciences Pvt Ltd. This is an open access article under the CC BY license (http://creativecommons.org/licenses/by/4.0/) DOI: http://dx.doi.org/10.22159/ijpps.2018v10i5.24311

\section{INTRODUCTION}

Development of fast disintegrating tablets (FDT) has gained wide interest in the last few years. These tablets are claimed to undergo fast disintegration in the mouth with rapid drug dissolution with subsequent absorption reaching the systemic circulation this dosage form is more convenient for children and elderly patients with swallowing difficulties [1]. However, the poor aqueous solubility of many drugs provide limited candidates for such a dosage form.

As a considerable number of newly developed chemical entities are classified as Class II drugs according to the biopharmaceutical classification system (BCS), meaning of low solubility with no permeability problems. Improvement of drug solubility and consequently its oral bioavailability remains of the utmost importance step in developing of new pharmaceutical product. There are several approaches reported in literature to enhance the solubility of poorly water-soluble drugs such as micronization [2], solid dispersion [3, 4], liquisolid tablet [5], self-emulsifying system $[6,7]$. These techniques were chosen on the basis of certain aspects such as properties of drug under consideration, nature of excipients to be selected, and nature of intended dosage form.

Though size reduction technique remains the most convenient and cost effective way for enhancing drug solubility, non-homogenous particle size distribution and possibleparticle agglomeration would decrease the surface available for dissolution $[8,9]$. On the other hand, an amorphous solid-state powder may improve the bioavailability of a slightly water soluble drug due to increased solubility and hence absorption of the drug in the gastrointestinal tract. However, the amorphous form usually suffer stability and hygroscopicity problems leading to conversion to a more stable crystalline form during storage.

In-situ micronizationcan be used as alternative to powder micronization. It is a relatively recent technique where micron sized crystals are obtained during its production without the need for any further particle size reduction. It is one of the easiest technique for producing micro crystals in one-step process that requires common equipment whereas other micronization techniques like milling, spray drying, and supercritical fluid require specialized equipment and containment facilities [10].

In situ crystallization approaches are generally based on the drug precipitation from a supersaturated solution of the drugusingantisolvent [11]. This technique produces crystals from solutions and can controls the crystalline properties $[12,13]$. Co-precipitation of the drug with a stabilizer would impart a hydrophilic surface to the crystals that would, otherwise, aid in the dissolution step. Addition of hydrophilic polymers during the precipitation step would act to stabilizecrystal germs against possible crystal growth behavior. Additionally, crystalline substances obtained by this technique showed less cohesiveness and better flow properties [10]. In situ controlled crystalization was previously used to enhance dissolution of many drugs, such as Carbamazepine [12], ketoconazole [10], Glibencalmide [13] and flurbiprofen [14].

Indomethacin is a non-steroidal anti-inflammatory drug (NSAID) commonly used as a prescription medication to reduce fever, pain, stiffness, and swelling. It works by inhibiting the production of prostaglandins. Indomethacin is classified as class II drug in the BCS, with poor aqueous solubility [15]. As a result of its bad solubility and erratic oral bioavailability, gastro-intestinal irritation was reported due to long contact time with the mucosa [16].

The objective of this work was to increase the dissolution rate of indomethacin with the aim of producing fast disintegrating tablets (FDT). This will be achieved by using in situ controlled crystallization technique. The effect of presence of stabilizer during the precipitation process on drug physical properties and dissolution behavior was also investigated. To find a stabilizer with high affinity to the newly created 
high surface area, several potential stabilizing agents were compared including hydrophilic polymers and non-ionic surfactant. This technique will provide a potential for large scale production of drug particles with improved aqueous solubility.

\section{MATERIALS AND METHODS}

Indomethacin and hydroxypropylmethyl cellulose (HPMC E5) were obtained as a gift samples from Sigma Pharmaceutical Company, Quesna, Egypt. Polyvinylpyrrolidone K40 (PVP K40) was purchased from Sigma Chemical Co., Steinheim, Germany. Polyethylene glycol 6000 (PEG 6000) was obtained from BDH chemical Ltd., Pool, England. Tween 80 was purchased from El Nasr Pharmaceutical chemicals Co., Cairo, Egypt. Glucire44/14 was obtained as a gift from Gattefosse Company, France.

\section{UV spectrophotometric assay of indomethacin}

The study employed a UV spectrophotometer (Thermo Fisher Scientific, Madison, USA). Stock solution of indomethacin $(1 \mathrm{mg} / \mathrm{ml})$ in ethanol was used to prepareserial dilutions to provide concentrations of $4,6,8,10,12,14$ and $16 \mu \mathrm{g} / \mathrm{ml}$. A standard curve of indomethacin was constructed. The absorbance values were measured spectrophotometrically at $\lambda$ max of $320 \mathrm{~nm}$. The standard curve was linear $\left(\mathrm{R}^{2}=0.996\right)$ over the range of concentrations used.

\section{Preparation of in situ indomethacin microcrystals}

The composition of the prepared formulations is presented in table 1 . The procedure was performedusing bottom-up approach that is generally based on the drug precipitation from a supersaturated solution of the drug usingde-solvent [17]. The precipitation process was conducted in presence or absence of hydrophilic polymer or nonionic surfactant. The selected surfactants were Tween 80 and Glucire 40/14, while polymers were HPMC E5, PEG 6000, and PVP K40. The drug: additive weight ratio was 1:1, except for Tween and Glucire where a weight ratio of 1:0.5 was used. Ternary mixture of HPMC E5 and Tween80was prepared (table 1). The drug was dissolved in the least amount of ethanol. The polymer or surfactant was dissolved in water and added to drug solution while stirring using magnetic stirrer. Drug crystals were obtained as white precipitant. The crystals were recovered after being centrifugedat $2000 \mathrm{rpm}$ for $10 \mathrm{~min}$ and stored in a desicator over silica gel at room temperature till complete dryingand were stored in a tightly closed container.

Table 1: Composition of different formulations, together with in vitro dissolution parameters represented as percentage drug released after 5 min (Q5) release efficiency (RE) in acidic and basic media

\begin{tabular}{|c|c|c|c|c|c|c|}
\hline \multirow[t]{2}{*}{ Formula } & \multirow{2}{*}{$\begin{array}{l}\text { Hydrophilic } \\
\text { additive }\end{array}$} & \multirow[t]{2}{*}{ Drug: polymer ratio } & \multicolumn{2}{|c|}{ Acidic media (pH 1.2) } & \multicolumn{2}{|c|}{ Basic media (pH 6.8) } \\
\hline & & & Q5 & Q5 & \%RE & $\%$ RE \\
\hline Pure indomethacin & - & - & $1.1(4, \pm 0.5)$ & $1.6(4, \pm 0.1)$ & $7.5(3, \pm 0.2)$ & $14.7(3, \pm 0.9)$ \\
\hline F1 & - & - & $1.2(3, \pm 0.2)$ & $2.2(3, \pm 0.2)$ & $18.8(3, \pm 0.6)$ & $25.7(3, \pm 2.7)$ \\
\hline F2 & Polyethylene glycol 6000 & $1: 1$ & $1.4(3, \pm 0.1)$ & $2.9(3, \pm 0.3)$ & $24.8(3, \pm 0.9)$ & $49.0(3, \pm 2.8)$ \\
\hline F3 & Hydroxypropylmethycellulose E5 & $1: 1$ & $8.5(3, \pm 1.1)$ & $12.0(3, \pm 0.8)$ & $33.63,( \pm 0.8)$ & $52.2(3, \pm 3.1)$ \\
\hline $\mathrm{F} 4$ & Polyvinylpyrrolidone $\mathrm{K} 40$ & $1: 1$ & $7.2(4 \pm 1.1)$ & $12.8(4, \pm 1.2)$ & $24.7(3, \pm 0.5)$ & $48.5(3, \pm 2.9)$ \\
\hline F5 & Tween 80 & $1: 0.5$ & $1.5(3, \pm 0.1)$ & $4.4(3, \pm 0.5)$ & $33.4(3, \pm 1.3)$ & $49.2(3, \pm 1.5)$ \\
\hline F6 & Glucire $40 / 14$ & $1: 0.5$ & $1.7(3, \pm 0.6)$ & $5.6(3, \pm 2.2)$ & $16.7(3, \pm 0.5)$ & $35.9(3, \pm 2.0)$ \\
\hline F7 & Ternary mix (drug: HPMC: Tween80) & $1: 1: 0.2$ & $1.5(4, \pm 0.3)$ & $4.8(4, \pm 0.8)$ & $49.3(4, \pm 1.0)$ & $76.6(4, \pm 1.9)$ \\
\hline
\end{tabular}

Values between brackets are number of replicates and standard deviation, respectively.

\section{Drug content}

The drug content was determined for the prepared crystals by dissolving an amount equivalent to $50 \mathrm{mg}$ of the drug from each formulation in a $50 \mathrm{ml}$ ethanol. After suitable dilution, drug concentration was determined spectrophotometrically at $320 \mathrm{~nm}$. The experiment was performed in triplicate and the drug content was expressed as $\mathrm{mg} / \mathrm{ml}$ concentration.

\section{Physical state characterization}

These studies were performed to investigate the physical properties of the drug after precipitation and to detect any possible interaction with other additives. Differential scanning calorimetry (DSC), X-ray diffraction and Fourier transform infrared spectroscopy (FTIR) were used to achieve this goal.

\section{Differential scanning calorimetry}

Unprocessed indomethacin, HPMC E5, PEG6000, PVP k40, and prepared crystals were studied regarding their thermal behavior using differential scanning calorimetry (DSC-60, Shimadzu, Japan). Aluminum pans loaded with samples equivalent to approximately 2 $\mathrm{mg}$ of the drug were crimped using a Shimadzucrimper. The thermal behavior of each sample was investigated at a heating rate of 10 ${ }^{\circ} \mathrm{C} / \mathrm{min}$, covering temperature ranges of $25-300{ }^{\circ} \mathrm{C}$. Data analysis was conducted using the TA-60WS thermal analysis software and the transition midpoint $\left(\mathrm{T}_{\mathrm{m}}\right)$ of the drug was recorded.

\section{X-ray powder diffraction}

Powder X-ray diffraction patterns were traced for unprocessed indomethacin and prepared formulations as well as pure polymers. The study utilized powder diffractometer (Crystal Impact, Bonn, Germany). Samples were loaded into aluminum containers. Scanning was performing datarate of $8 \%$ min over a 2 theta $(2 \theta)$ range from 3 to 60 .

\section{Fourier transform infrared spectroscopy}

The Fourier transform infrared (FTIR) spectra of pure indomethacin, pure polymers and prepared microcrystal formulations were recorded using FTIR spectrophotometer (FTIR-Spectrometer, Tensor 27, Bruker, USA). Disks of potassium bromide and tested sample mixtures were obtained using hydraulic press before scanning at a range of 4000 through $600 \mathrm{~cm}^{-1}$.

Preparation and characterization of fast disintegrating tablets (FDT)

The precipitated crystals showing the best dissolution parameters was selected to prepare fast disintegrating tablets by direct compression technique. This process employed single punch tablet machine (Royal Artist, Kapadia Industrial Estate, BLDG, Mumbai, India). The compression force was adjusted to produce tablets having a hardness in the range of 4-5 KP.

Each tablet was prepared to contain an amount equivalent to $50 \mathrm{mg}$ of the drug. The inactive components of tablets were granular mannitol, Avicel PH101, Ludiflash $\AA$, crospovidone and magnesium stearate in the concentration of $75 \mathrm{mg}, 50 \mathrm{mg}, 12 \mathrm{mg}, 12 \mathrm{mg}$ and 5 mg per tablet, respectively. Mannitolasfillerthatimproves taste due to its mildsweet taste in addition to its known cooling sensation due to its negative heat of dissolution. Avicel PH101as directly compressible filler. Ludiflash ${ }^{\circledR}$ is a novel super-disintegrant.

\section{Evaluation of FDT}

The prepared tablets were evaluated regarding drug content uniformity, weight variation, disintegration time and dissolution rate. Tests procedure and limits of acceptance were in accordance to United States Pharmacopeia National Formulary 2000 [18].

Additionally, the wetting time of the tablets was also determined. The test was performed in petri-dish containing wetted filter paper with $6 \mathrm{ml}$ of distilled water. A small amount of Allura red powder 
was placed on the top surface of each tablet before placing over the wet filter paper. The time required for developing a red color on the surface was recorded and taken as the wetting time [19].

\section{In vitro release studies}

The dissolution rate of indomethacin from different in situ microcrystals was determined using the USP II dissolution apparatus (Copley, NG 42 JY, Nottingham, UK). Raw indomethacin was used as control. Indomethacindis solution was evaluated both in acidic and basic dissolution medium. The acidic medium was $0.1 \mathrm{~N} \mathrm{HCl}(\mathrm{pH} 1.2)$ simulating gastric condition, and the alkaline medium was phosphate buffer $\mathrm{pH} 6.8$ containing $0.15 \%$ sodium dodecylsulfate simulating intestinal conditions. The paddle speed was adjusted to $100 \mathrm{rpm}$ and dissolution medium $(900 \mathrm{ml})$ was maintained at $37{ }^{\circ} \mathrm{C}$. Following loading the drug $(50 \mathrm{mg}$ ) or its equivalent from crystal formulations, samples of $5 \mathrm{ml}$ each were withdrawn at a predetermined time interval for a length of $60 \mathrm{~min}$, and were replaced with fresh dissolution medium. Fast dissolving tablets were tested in the buffer solution using the same dissolution conditions. After filtering through $0.45 \mathrm{~mm}$ Whatman membrane filter, each sample was analyzed spectrophotometricaly for drug content. The cumulative amounts drug dissolved (expressed as \% of the total drug load) were plotted as a function of time to obtain the dissolution profiles. Dissolution parameters were calculated and compared.

\section{Statistical analysis}

All experiments were conducted in triplicates and Statistical analysis employed Student $t$-test. Results were quoted as significant when $P<0.05$.

\section{RESULTS AND DISCUSSION}

\section{Solid state characterization of the prepared microcrystals}

Drug content of the prepared crystals were in the range of $97-99 \%$ $\mathrm{w} / \mathrm{w}$, excluding any possibility for segregation of drug or additives during preparation (table 1 ).

\section{Differential scanning calorimetry (DSC)}

DSC of pure indomethacin and different formulations are shown in fig. 1. High phase transition temperature $\left(\mathrm{T}_{\mathrm{m}}\right)$ and peak sharpening would indicate high degree of crystalline structure of a substance. Peak shifting to a lower $\mathrm{T}_{\mathrm{m}}$ and/or broadening would designate reduced drug crystallinity [19]. Unprocessed indomethacin showed a characteristic endothermic peak with a $\mathrm{T}_{\mathrm{m}}$ beingrecorded at $161{ }^{\circ} \mathrm{C}$ indicating the crystalline nature of the drug (fig. 1A). The recorded endothermic peak correlates well with values published by other investigators $[20,21]$. Thermogram for pure PEG 6000 showed a sharp endothermic peak with $\mathrm{T}_{\mathrm{m}}$ of about $60^{\circ} \mathrm{C}$ (fig. $\left.1 \mathrm{~A}\right)$, agreeing with reported transition behavior of the same polymer [13]. For pure HPMC the thermal event showed a broad endotherm with onset of $39.2^{\circ} \mathrm{C}$ with an end set of $80.1^{\circ} \mathrm{C}$. Similarly, PVP thermogram recorded a broad endothermic peak with onset of $30.9^{\circ} \mathrm{C}$ and endset of $81.0{ }^{\circ} \mathrm{C}$. These broaden peaks represent the desorption of the bound water from such hygroscopic materials [14, 22, 23].

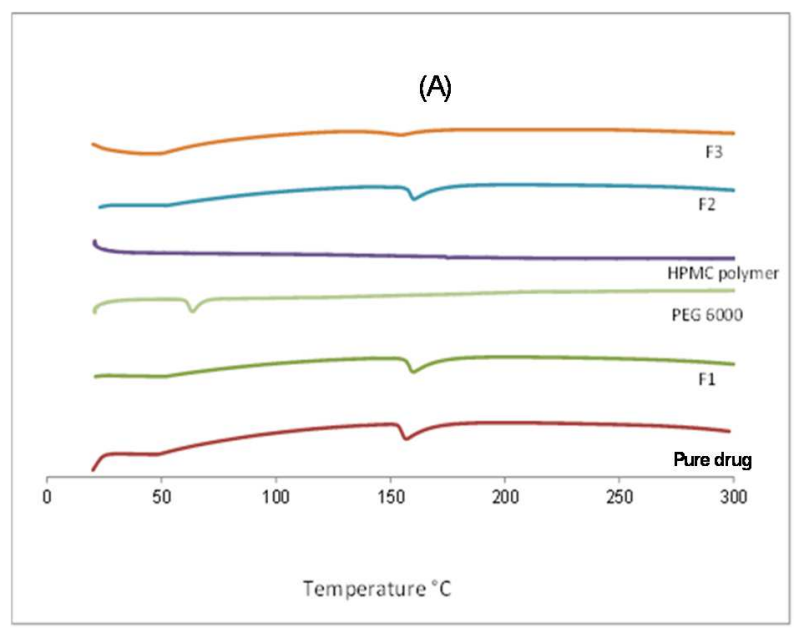

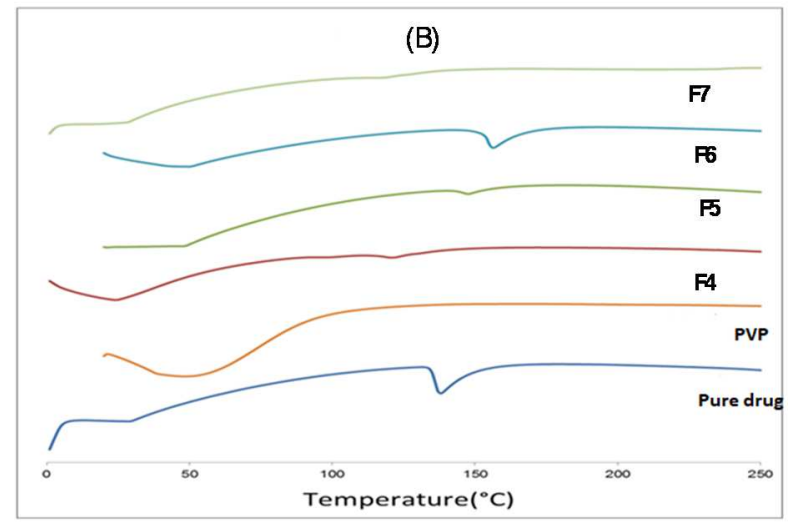

Fig. 1: Examples of DSC traces of pure indomethacin, polyethylene glycol 6000 (PEG 6000), hydroxypropyl methyl cellulose E5 (HPMC), polyvinylpyrrolidone (PVP) and different formulations. For detailed formulations refer to table 1

Regarding plain indomethacin crystals (F1, in situ crystals without polymer) the endothermic peak can be detected at the same $\mathrm{T}_{\mathrm{m}}$ with reduced sharpness and slight peak broadening (fig. 1A). This would suggest possible reduction in crystal size during the in situ crystallization. Regarding the prepared crystals in presence of hydrophilic additives, all formulations showed a slight shift in $\mathrm{T}_{\mathrm{m}}$ to a lower value compared to that without polymer and unprocessed drug. Presence of surfactants during in situ crystallization (Tween 80 "F2" and Glucire 44/14 "F6") resulted in slight reduction in transition temperature with $\mathrm{T}_{\mathrm{m}}$ appearing at $159^{\circ} \mathrm{C}$ (fig. $2 \mathrm{~A}$ and $\mathrm{B}$ ). This reduction accompanied by peak broadening with a slight reduction in peak area and enthalpy.

In situ precipitation in presence of polymer showed considerable change in thermal behavior different from that of unprocessed drug and polymer. For F3, prepared using PEG 6000, thermogram showed broad symmetric endothermic peak at $159.1^{\circ} \mathrm{C}$ with an onset of 145 ${ }^{\circ} \mathrm{C}$ and endset of $175^{\circ} \mathrm{C}$ (fig. 1A). For F5, prepared using PVP, similar thermal pattern was observed with melting transition of the drug appearing at $158.5^{\circ} \mathrm{C}$ (fig. 1B).

For F4, prepared using HPMC with a Tm of $143{ }^{\circ} \mathrm{C}$ considerable peak broadening and reduced enthalpy. The maximum effect was observed for formula F7, precipitated drug in presence of both HPMC and Tween 80, where the endothermic peak underwent significant broadening with the melting transition of drug is almost abolished. This thermal pattern suggests reduced particle size of drug crystals. Additionally, transformation of the drug into amorphous form with probable modification of the crystalline structure is also a possibility $[14,20]$. This requires further confirmation by with other instrumental techniques.

\section{X-ray powder diffraction (XRPD)}

The diffractograms of pure drug, polymers and different formulations are presented in fig. 3. The diffraction pattern of unprocessed drug showed numerous diffraction peaks at angle (20) of $9^{\circ}, 11.5^{\circ}, 16.8^{\circ}, 19.6^{\circ}, 21.7^{\circ}$ and $26.6^{\circ}$. This reflects the crystalline nature of the drug. This diffraction pattern is similar to reported data for the same drug and indicates that majority of indomethacin is present in itsgamma form [20-23]. In situ crystallization of the drug in presence or absence of hydrophilic polymers and/or surfactant resulted in diffractograms with diffraction peaks at the same position of those recorded in case of the pure drug but with lower intensity (fig. 2). It worth noting that the reduction in peak intensity was higher for crystals precipitated in presence of the hydrophilic additives. This verifies that a considerable amount of drugispresent in crystalline state in addition to the amorphous form. Meantime, possible reduction in the particle size after in situ crystallization can also be considered. Reduced intensity of the diffraction peaks was previously explained as an indication of reduced particle size [14, 24]. The highest reduction in peak intensity was noticed for crystals prepared in presence of 
HPMC and Tween80 (F7). Thisfindingis in good agreement with DSC data. It is important to highlight that the reduced peak intensity may indicate the possible partial formation of amorphous form during the recrystallization step.
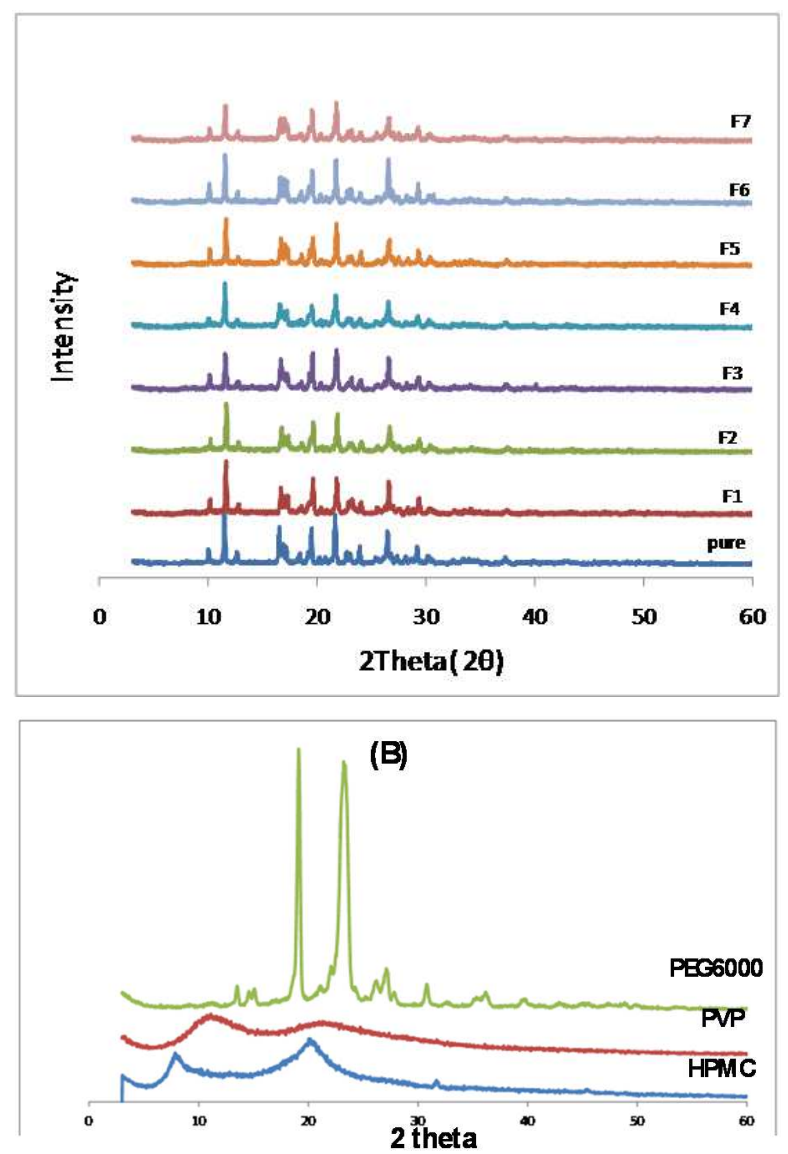

Fig. 2: X-ray diffractograms of (A) pure indomethacin and different formulations (B) pure polyethylene glycol 6000 (PEG 6000), hydroxypropylmethyl cellulose (HPMC) and polyvinylpyrrolidone (PVP). For detailed formulations refer to table 1

\section{Fourier transform infrared spectroscopy (FTIR)}

The infrared study was performed to examine any possible interaction between pure drug and additives. Fig. 3 shows the FTIR spectra of indomethacin, pure polymers and the prepared formulations.

Pure indomethacin spectra showed characteristic peaks represented as aromatic C-H stretching at $3020 \mathrm{~cm}^{-1}, \mathrm{C}-\mathrm{H}$ stretching vibrations at $2965 \mathrm{~cm}^{-1}, \mathrm{C}=0$ stretching vibrations shown at $1760 \mathrm{~cm}^{-1}$. Peak at $1599 \mathrm{~cm}^{-1}$ for aromatic $\mathrm{c} \equiv \mathrm{c}$ stretching. The asymmetric aromatic $\mathrm{O}-\mathrm{C}$ stretching reveals at $1261 \mathrm{~cm}^{-1}$ and that for symmetric aromatic $0-\mathrm{H}$ stretching vibration at $1086 \mathrm{~cm}^{-1}$. This spectra in good agreement with published data [21].

The FTIR spectrum of pure PVP K40 was characterized by its carbonyl group which appeared at lower frequency of $1655 \mathrm{~cm}^{-1}$ due to hydrogen bonding with the adsorbed water. There was a broad band at $3429 \mathrm{~cm}^{-1}$ for the hydrogen bonded $\mathrm{OH}$ group indicating hygroscopic property of PVP [26]. For HPMC, a broad band of hydroxyl group was shown in the range of $3100-3600 \mathrm{~cm}^{-1}$. This agrees with the published spectrum of the same polymer [26, 27]. The spectrum of PEG 6000 reveals broad band at $3446 \mathrm{~cm}^{-1}, 2887$ $\mathrm{cm}^{-1}, 1113 \mathrm{~cm}^{-1}$ for the $\mathrm{OH}$ group, aliphatic $\mathrm{C}-\mathrm{H}$ stretching and $\mathrm{C}-\mathrm{O}$ stretching, respectively. This finding is in good correlation with the previous published data of the same polymer [27]. FTIR spectrums of tested formulations revealed the main absorption bands of indomethacinwith no significant changes compared with the spectrum of pure drug. This would suggest absence of any possible interaction between the drug and polymers.
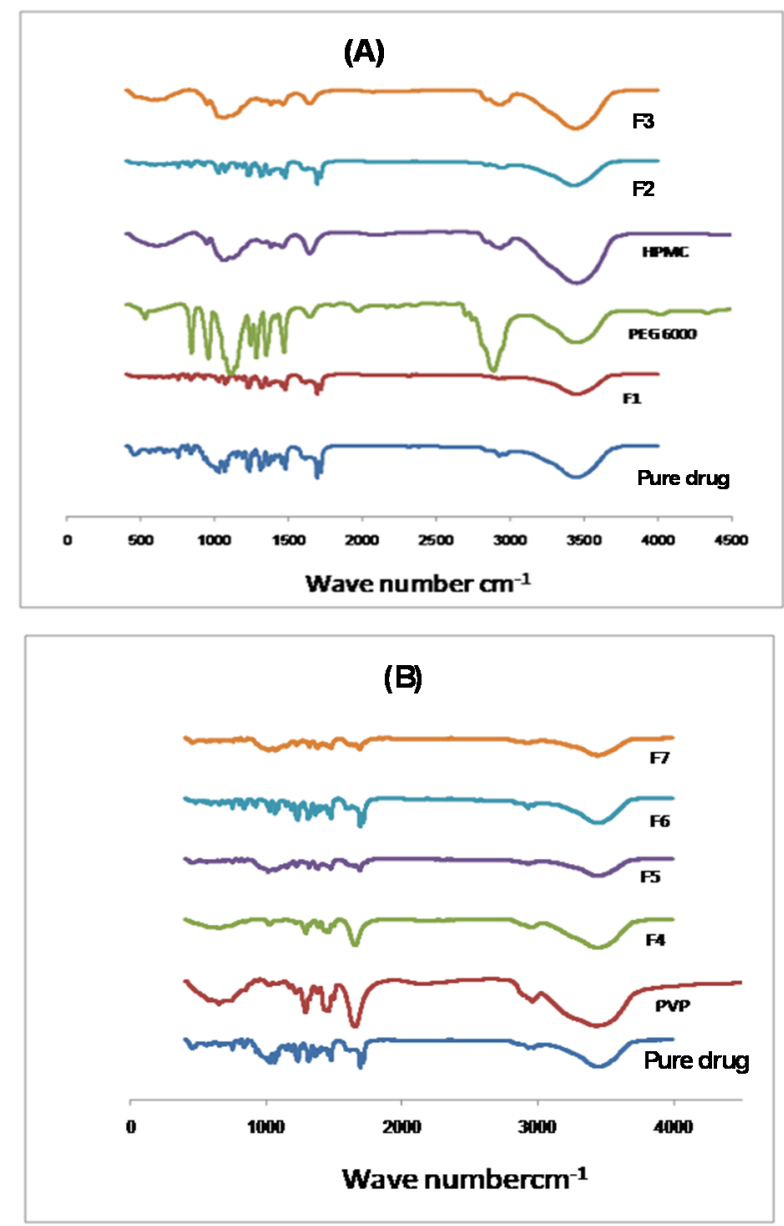

Fig. 3: FTIR spectra of unprocessed indomethacin and different formulations together with pure polyethylene glycol (PEG 6000), hydroxypropyl methyl cellulose (HPMC), and polyvinylpyrrolidone (PVP)) together. For detailed formulations refer to table 1

\section{In vitro drug release studies}

The dissolution profiles of indomethacin from the rawpowderand powders prepared by in situ controlled crystallization in presence of different hydrophilic additives are presented as percentage drug released versus time plots in fig. 4 . The dissolution parameters were extracted from the profiles and represented as the percentage amountofdrug released after $5 \mathrm{~min}\left(\mathrm{Q}_{5}\right)$ in acid and basic conditions. The percentage release efficiencies (RE) in both media were calculated from the area under the dissolution profile of each formulation at time $t$ and expressed as the percentage of the area of the rectangle described by $100 \%$ dissolution in the same time [28]. These parameters are presented in table 1.

In acidic medium, unprocessed drug showedvery slow drug dissolution with a Q5 of $1 \%$ and a RE of about $2 \%$. This poor dissolution can be attribute to the hydrophobic nature of the drug as well as the reported limited solubility in acidic conditions [29]. Preparation of plain drug crystals slightly improved drug release compare to control (fig. 4A and table 1). The in situ crystallization of indomethacin in presence of hydrophilic polymers and/or surfactant was studied with the aim to impart hydrophilic characters to the crystal surface and to reduce the possible particles aggregation up on storage. PVP k40, HPMC E5 and PEG 6000 were selected as the 
hydrophilic polymers, while Tween 80 and Glucire 44/14 was used as an example for nonionic surfactants according to the formulations presented in table 1 . Additionally ternary mixture of drug, HPMC and Tween 80 was also investigated.

In situ crystals prepared in presence of hydrophilic polymer or surfactant showed a trend of increased Q5 and RE, except for F4 and F5 prepared in presence of HPMC and PVP, respectively. The latter two formulations showed the highest significant increased dissolution parameters $(\mathrm{P}<0.05)$ with about 7 -fold and 12 -fold enhancement in Q5and RE, respectively.
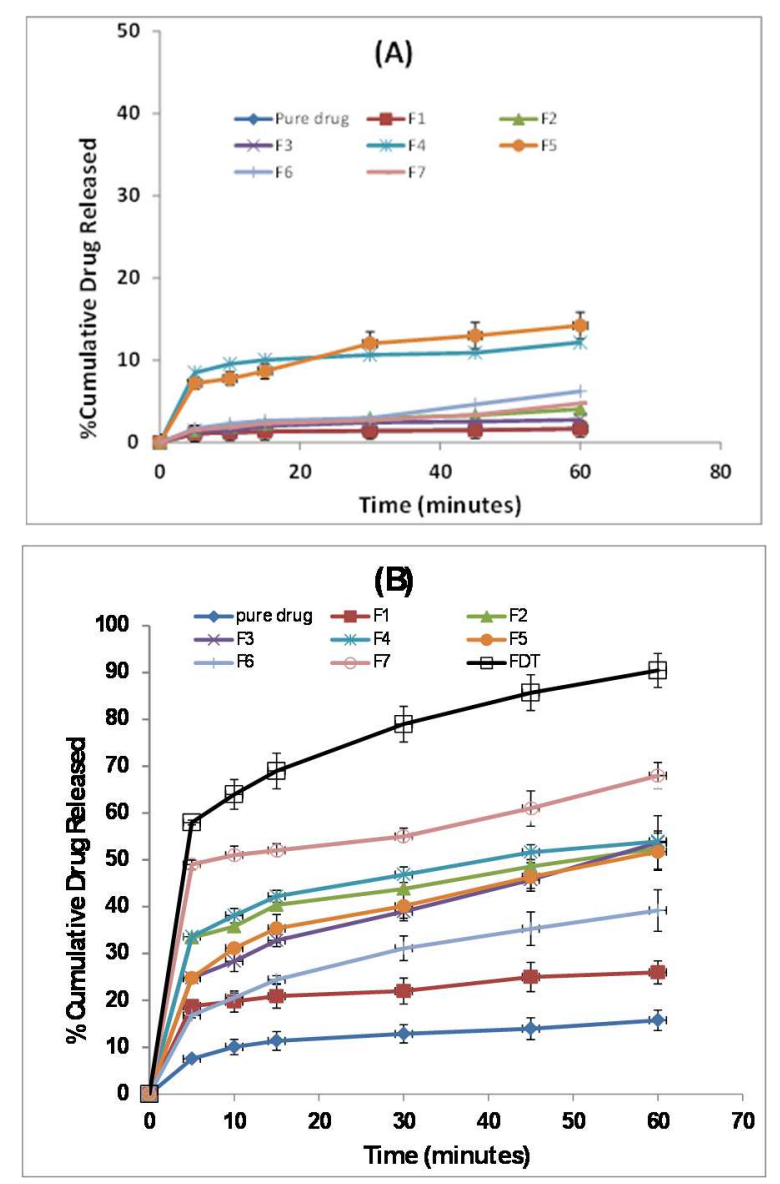

Fig. 4: In vitro drug dissolution of indomethacin from unprocessed form and different drug crystal formulationsin acidic (A) and basic (B) media, together with in vitro dissolution of fast disintegrating tablets $(B)(n=3)$

Contrary to acidic environment, dissolution of pure indomethacin and prepared formulations in alkaline media $\mathrm{pH}$ 6.8) showed a better dissolution behavior. Unprocessed drug showed Q5 and RE of about 7.5 and $15 \%$, respectively. Plain indomethacin microcrystals (F1) showed a similar Q5 value to that of control; however 2-fold enhancement in release efficiency was noted. This result could be due to decreased particle size during the precipitation step with subsequent increase in the surface area, as evidenced by DSC and xray results. Partial change in crystalline nature of the drug is also a possible explanation.

All formulations prepared in presence of hydrophilic additives showed a considerable increase in dissolution parameters over unprocessed drug (fig. 4A and table 1). There was a significant increasein $Q_{5}$ and $\mathrm{RE}$ relative to pure drug $(\mathrm{P}<0.05)$. The ternary mixture of indomethacin, HPMC and Tween 80 (F7) showed the highest dissolution parameters with a Q5 and RE of $49 \%$ and $76 \%$, respectively. The superiority of formulations prepared by in situ crystallization of the drug in presence of hydrophilic substance could be due to the possible adsorption of the hydrophilic molecules on the microstructure of the crystal surface during the precipitation and separation processes [14]. Such adsorption could be due to polar and nonpolar interactions of the hydrophilic molecules and drug such as Van der Waals, hydrogen bonding and/or hydrophobic interaction [30]. Such interaction between the hydrophilic additive and the surface of the newly formed crystals will improve the crystal behavior by two possible mechanisms. Firstly, increasing the hydrophilicity and consequently wettability of drug particles. Secondary, sterically stabilizing crystals against agglomeration because of their hydrophobic surface. Therefore, all these factors (increased surface wettabilityand reduced crystal size) would act synergistically. For the ternary system in F7, presence of hydrophilic polymer HPMC and surfactant resulted in augmented effect in enhancing drug dissolution.

The overall results thus reflect the potential of the controlled precipitation technique as a tool to improve drug dissolution behavior by proper manipulation of polymer type and concentration. Formula F7, showed best dissolution parameters, was selected to prepare fast disintegrated tablets.

\section{Characterization of fast disintegration tablets}

Regarding the quality control studies, tablets complied with the US Pharmacopeia requirements for acceptable tablets batch. For the weight variation, the deviation from the mean tablet weight was $<2 \%$ as a reflection of good powder flowability. The recorded content uniformity values were in the range of $97 \pm 2.6 \%$, the friability was found to be $0.9 \%$, indicating acceptable resistance of tablets to handling. The wetting time was $30 \pm 0.2$ secondsThis short wetting time could be due to reduced hydrophobicity of the drug after controlled precipitation in presence of hydrophilic polymers. The average disintegration time of 50 second was noted and can be attributed to the presence of super-disintegrant, Ludiflash ${ }^{\circledR}$, that aids in rapid tablet break down.

The dissolution profile for FDT is presented in fig. 5B. Tablets exhibited a relatively better dissolution behavior compared with the unprocessed drug powder. This is reflected by recorded $Q_{5}$ and RE of $58 \%$ and $83 \%$, respectively. The enhanced dissolution of indomethacin from FDT compared to the F7 powder, optimized formula used in tablet preparation, can be explained by the adsorption of the drug on the surface of tablet excipients with subsequent rapid dispersion in the dissolution medium. This also proof that the compression force used during tablet manufacturing didn't affect the dissolution rate of the obtained drug crystals.

\section{CONCLUSION}

In situ controlled crystallization in presence of hydrophilic polymer and/or surfactants is a useful tool to enhance dissolution rate of indomethacin. Such enhancement, particularly in the basic media, can be attributed to adsorption of hydrophilic substance over drug particle. Reduced particle size after precipitation can provide another explanation for such improvement. Partial formation of amorphous drug particles is also a possibility. The optimized ternary drug/HPMC/Tween80 mixture, showing the best dissolution parameters, was successfully formulated as fast disintegrating tablets with subsequent rapid drug release.

\section{AUTHORS CONTRIBUTION}

All authors had equally contributed the research work

\section{CONFLICTS OF INTERESTS}

All authors have none to declare

\section{REFERENCES}

1. Shailendra K, Dina NM, Rishab J, Pankaj S. Fast disintegrating combination tablets of omeprazole and domperidone. Asian J Pharm Clin Res 2009;2:74-82.

2. Vogt M, Kunath K, Dressman JB. Dissolution enhancement offenofibrate by micronization, cogrinding and spray-drying: comparisonwith commercial preparations. Eur J Pharm Biopharm 2008;68:283-8. 
3. Kulkarani U, Patil BS, Hariprasanna RC, Borgaonkar PA, Hogade MG. Formulation and development of fast dissolving meloxicam tablets by solid dispersion technique. Int J Curr Pharm Res 2010;2:82-5.

4. Essa EA. Enhancement of carvedilol dissolution: surface solid dispersion versus solid dispersion. Asian J Pharm 2015;8: 283-9.

5. Elkordy AA, Tan XN, Essa EA. Spironolactone release from liquisolid formulations prepared with Capryol ${ }^{\mathrm{TM}}$ 90, Solutol® HS-15 and Kollicoat® SR 30 D as Non-volatile liquid vehicles. Eur J Pharm Biopharm 2013;83:203-23.

6. Saurabh SS, Issarani R, Nagori BP. Formulation and evaluation of self-emulsifying drug delivery system of etoricoxib. Asian J Pharm Clin Res 2017;10:367-72.

7. Balata GF, Essa EA, Shamardl HA, Zaidan SH, Abourehab MAS. self-emulsifying drug delivery systems as a tool to improve solubility and bioavailability of resveratrol. Drug Des Dev Ther 2016;10:117-28.

8. Sunday AS, Simon C. Particle engineering techniques for inhaled biopharmaceuticals. Adv Drug Delivery Rev 2006;58:1009-29.

9. Yohei K, Koichi W, Manabu N, Shizuo Y, Satomi O. Formulation design for poorly water-soluble drugs based on biopharmaceutics classification system: basic approaches and practical applications. Int J Pharm 2011;420:1-10.

10. Rasenack N, Muller BW. Dissolution rate enhancement by in situ micronization of poorly water-soluble drugs. Pharm Res 2002;19:1894-900.

11. Xia D, Quan P, Piao H, Piao H, Sun S, Yin Y, et al. Preparation of stable nitrendipine nanosuspensions uing the precipitationultrasonication method for enhancement of dissolution and oral bioavailability. Eur J Pharm Sci 2010;40:325-34.

12. Park MW, Yeo SD. Antisolvent crystallization of carbamazepine from organic solutions. Chem Eng Res Design 2012;90:2202-8.

13. El Kordy AA, Jatto A, Essa EA. In situ controlled crystallization as a tool to improve the dissolution of glibenclamide. Int J Pharm 2012;428:118-20.

14. Essa EA, Elmarakby A, Donia A, El Maghraby GM. Controlled precipitation for enhanced dissolution rate of flurbiprofen: development of rapidly disintegrating tablets. Drug Dev Ind Pharm 2017;24:1-10.

15. Lobenberg R, Amidon GL. Modern bioavailability: bioequivalence and biopharmaceutics classification system: new scientific approaches to international regulatory standard. Eur J Pharm Biopharm 2000;50:3-12.
16. Al Saidan SM, Alsughayer A, Eshra AG. Improved dissolution rate of indomethacin by adsorbents. Drug Dev Ind Pharm 1998;24:389-94.

17. Sinha B, Muller RH, Moeschwitzer JP. Bottom Up approaches for preparing drug nanocrystals: formulation and factors affecting particle size. Int J Pharm 2013;453:126-41.

18. United States Pharmacopiae National Formulary 24, United States Pharmacopial Convention, Rockville, MD; 2000.

19. Jain CP, Naruka PS. Formulation and evaluation of fast dissolving tablets of valsartan. Int J Pharm Pharm Sci 2009;1:219-26.

20. Bettinetti GP, Sorrenti M, Rossi S, Ferrari F, Mura P, Faucci MT. Assessment of solid-state interactions of naproxen with amorphous cyclodextrin derivatives by DSC. J Pharm Biomed Anal 2002;30:1173-9.

21. Otsuka M, Kato F, Matsuda Y, Ozaki Y. Comparative determination of polymorphs of indomethacin in powders and tablets by chemometrical near-infrared spectroscopy and x-ray powder diffractometry. AAPS PharmSciTech 2003;4:1-12.

22. Balata G, Shamardl H. Controlled crystallization as a tool to enhance the dissolution and anti-inflammatory properties of indomethacin. J Pharm Res 2012;5:4175-81.

23. El-Badry M, Fetih G, Fathy M. Improvement of solubility and dissolution rate of indomethacin by solid dispersions in gelucire 50/13 and PEG4000. Saudi Pharm J 2009;17:217-25.

24. Arafa MF, El-Gizawy SA, Osman MA, El Maghraby GM. Sucralose as Co-Crystal Co-former for hydrochlorothiazide: development of oral disintegrating tablets. Drug Delivery Ind Pharm 2016;42:1225-33.

25. Shirke SH, Shete AS, Doijad RC. Enhancement of dissolution rate of indomethacin by Kollicoat IR based solid dispersions. Der Pharm Lett 2015; 7:64-73.

26. Essa EA, Balata GF. Preparation and characterization of domperidone solid dispersion. Pak J Pharm Sci 2012;25:783-91.

27. El Maghraby GM, Alomrani AH. Synergistic enhancement of itraconazole dissolution by ternary system formulation with pluronic F68 and hydroxypropylmethyl cellulose. Sci Pharm 2009;77:401-17.

28. Khan KA. The concept of dissolution efficiency. J Pharm Pharmacol 1975;27:48-59.

29. Nokhodchi A, Javadzadeh Y, Siahi-Shadbad MR, Barzegar-Jalali M. The effect of type and concentration of vehicles on the dissolution rate of poorly soluble drug indomethacin from liquisolidcompacts. J Pharm Pharmacol Sci 2005;8:18-25.

30. Wen H, Morris KR, Park K. Study on the interactions between polyvinylpyrrolidone (PVP) and acetaminophen crystals: partial dissolution pattern change. J Pharm Sci 2005;94:2166-74. 